\title{
Unique Aberrations in Intimal Sarcoma Identified by Next-Generation Sequencing as Potential Therapy Targets
}

\author{
Jason Roszik ${ }^{1, *,+} \mathbb{D}$, Abir Khan ${ }^{2,+}$, Anthony P. Conley ${ }^{3}$, J. Andrew Livingston ${ }^{3} \mathbb{D}$, \\ Roman Groisberg ${ }^{4}\left(\mathbb{D}\right.$, Vinod Ravi ${ }^{3}$, Roberto Carmagnani Pestana ${ }^{2}$, Shiraj Sen ${ }^{2,5}$ \\ and Vivek Subbiah $2, *$ (D) \\ 1 Department of Genomic Medicine, Division of Cancer Medicine, \\ The University of Texas MD Anderson Cancer Center, 1515 Holcombe Blvd., Houston, TX 77030, USA \\ 2 Department of Investigational Cancer Therapeutics, Division of Cancer Medicine, \\ The University of Texas MD Anderson Cancer Center, 1515 Holcombe Blvd., Houston, TX 77030, USA \\ 3 Department of Sarcoma Medical Oncology, Division of Cancer Medicine, \\ The University of Texas MD Anderson Cancer Center, 1515 Holcombe Blvd., Houston, TX 77030, USA \\ 4 Department of Sarcoma/Melanoma Medical Oncology, Rutgers Cancer Institute of New Jersey, \\ 195 Little Albany St, New Brunswick, NJ 08903, USA \\ 5 Sarah Cannon Research Institute, Denver, CO 80218, USA \\ * Correspondence: jroszik@mdanderson.org (J.R.); vsubbiah@mdanderson.org (V.S.); \\ Tel.: +1-713-745-2641 (J.R.); +1-713-563-1930 (V.S.); Fax: +1-713-745-1046 (J.R.); +1-713-792-0334 (V.S.) \\ + These authors contributed equally to this study.
}

Received: 24 July 2019; Accepted: 26 August 2019; Published: 31 August 2019

\begin{abstract}
Intimal sarcomas are rare and histologically heterogeneous tumors, commonly arising from the pulmonary arteries. They have remained challenging to treat. Few studies in the literature study the genomics of this cancer. Identifying targetable alterations is an important step in advancing the treatment of intimal sarcomas. Using data from the American Association for Cancer Research Project Genomics Evidence Neoplasia Information Exchange (AACR GENIE) database, we cataloged genetic alterations and assessed their clinical utility from thirteen patients with intimal sarcoma. Notable copy number alterations included amplification in MDM2, CDK4, PDGFRA, and NOTCH2, as well as copy number losses in CDKN2A and CDKN2B. Actionable alterations included mutations in ATM/ATR, PTCH1, and PDGFRB. Moreover, genomic rearrangement events, specifically PDE4DIP-NOTCH2 and MRPS30-ARID2 fusions were identified. Co-occurring alterations included a NOTCH2 copy number gain in the PDE4DIP-NOTCH2 fusion positive tumor and PDGFRB mutations in both fusion-positive cases. Our study suggests that PDGFRB may be relevant in the tumorigenesis process. Including genomic profiling in the management of intimal sarcoma and potential enrollment in targeted therapy trials is warranted.
\end{abstract}

Keywords: intimal sarcoma; next-generation sequencing; copy number alteration; somatic mutation; gene fusion; AACR GENIE

\section{Introduction}

Intimal sarcomas (IS) are rare mesenchymal tumors that involve the innermost layer of large vessels, most commonly seen in pulmonary arteries [1-4]. Diagnosing this sarcoma has proved challenging despite the criteria set by the World Health Organization (WHO), as it is largely undifferentiated and histologically variable even within the same host $[1,2,5-7]$. This tumor has the ability to differentiate into other types of sarcomas such as leiomyosarcoma and angiosarcoma $[1,3,5]$. Since intimal sarcomas 
are found post-operatively or at autopsy, the true incidence of this disease entity is unknown and likely underestimated [8]. Clinical presentation can be non-specific and variable, but these tumors can mimic signs and symptoms of thromboembolism which delays diagnosis [3,5,9]. The clinical course is often aggressive, with over $50 \%$ of patients having metastasis upon diagnosis $[5,10]$. There are no standardized treatments or protocols for this type of cancer [11]. Intimal sarcoma has been shown to be highly resistant to conventional chemotherapies such as anthracyclines, topoisomerase inhibitors, and alkylating agents [5,11-14]. Prognosis is poor, with a median survival of 12-13 months with radical surgery aimed at complete resection $[2,5,11]$. The challenging dynamic on a clinical and histologic level necessitates a comprehensive genetic approach to diagnose and develop targeted treatment plans. Unfortunately, limited data is available on the molecular characteristics of this tumor.

Attempts to elucidate the genomic profile of this rare tumor have been accomplished by only a few studies, but have emphasized the involvement of PDGFRA, a gene encoding tyrosine kinase receptors for platelet-derived growth factor. A case report on cardiac intimal sarcoma found copy gains in PDGFRA, KIT, STAT6, GLI-1, CKD4, HMGA2, and MDM2 (a negative regulator of p53) using whole exome sequencing (WES) and array-comparative genomic hybridization (aCGH) [4]. They also discovered concurrent PDGFR $\alpha$ amplification and PDGFR $\beta$ R709H mutation in intimal sarcoma [4]. The largest study of cardiac sarcomas analyzed tissue samples from 100 cases, and found intimal sarcomas to be the most frequent subtype of cardiac sarcoma, with amplification of MDM2 in all cases. They also identified copy gains of CDK4, HMGA2, DDIT3, and GLI coinciding with 12q12-15 region via immunohistochemical analysis and confirmed with fluorescence in situ hybridization (FISH), real-time polymerase chain reaction (RT PCR), and aCGH. Other findings included copy gains in 4q12 with KIT and PDGFRA, 7p12 with EGFR, and copy number loss of CDKN2A in 9p21 [1]. Another study of IS, which was the first to propose PDGFRA as a molecular hallmark for intimal sarcomas, found copy number gains of PDGFRA, EGFR, and MDM2 in 8 patients using FISH and aCGH with consistent activation of PDGFR and EGFR confirmed by western blotting [2]. Interestingly, a case report of IS in the abdominal aorta found intratumoral heterogeneity of PDGFRA amplification [7]. The tumor cells with the lowest degree of atypia, usually at the site of origin of the tumor, were not associated with overexpression of PDGFRA. Conversely, cells with the most atypia and aberrancy were found to have over-amplification of PDGFRA, suggesting PDGFRA is needed for tumorigenesis and the biopsy site is crucial for molecular studies [7]. Overall, these studies found recurrent amplification of PDGFRA, $P D G F R B, C K D 4$, and MDM2, implicating their involvement in the tumorigenesis process. No study has found somatic mutations leading to overexpression, or unique genetic fusions. Thus, the mechanism by which these genes are overexpressed is likely to be through gene amplification [1,2,12,15].

Other malignancies with dysregulated PDGFRA signaling, such as gastrointestinal stromal tumors (GIST), have received treatment with tyrosine kinase inhibitors (TKI) [16]. Ex vivo assays performed on tumor cell cultures exposed to a TKI for two hours from a single patient case with IS, demonstrated a dose-response in decreasing PDGFRA phosphorylation confirmed by western blotting [2]. One case series had 4 of 13 patients with IS initiated on a TKI, but given the molecular complexity of the tumor, remission was not achieved [5]. Unfortunately, as these tumors involve major vessels they are excluded from any potential early phase clinical trials.

\section{Results}

To identify common and unique genetic alterations in IS for targeted therapy, we queried data from the American Association for Cancer Research (AACR) Genomics Evidence Neoplasia Information Exchange (GENIE) genomic database and cataloged and assessed genomic alterations and their clinical utility. As shown in Figure 1, intimal sarcoma comprises only $0.6 \%$ of all sarcomas in this large database [17]. Among the 13 patients analyzed with intimal sarcoma, the median age was 46 (range 18-76); eight were female (61\%) (see Table 1). Genomics were performed on tissue from the primary tumor in ten patients and metastatic site in three patients. 

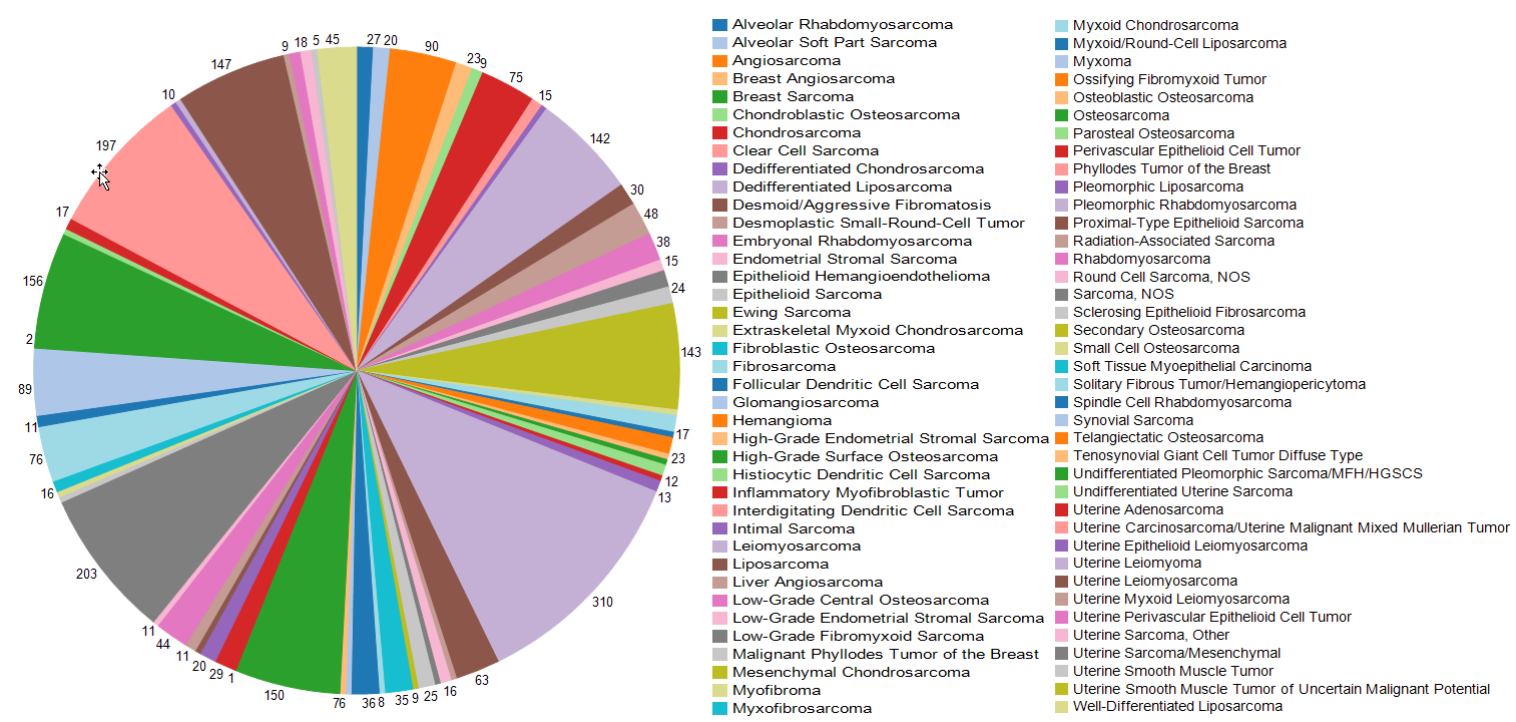

Figure 1. Sarcoma sample counts in American Association for Cancer Research Genomics Evidence Neoplasia Information Exchange (AACR GENIE). Color represents sarcoma subtypes as shown in the legend.

Table 1. Patient characteristics of the AACR GENIE intimal sarcoma patients.

\begin{tabular}{|c|c|c|c|c|c|c|}
\hline Patient & $\begin{array}{c}\text { Cancer Type } \\
\text { Detailed }\end{array}$ & Sample Type Detailed & $\begin{array}{l}\text { Age at Seq } \\
\text { Report }\end{array}$ & Sex & $\begin{array}{l}\text { Primary } \\
\text { Race }\end{array}$ & Ethnicity \\
\hline Patient 1 & Intimal Sarcoma & Metastasis site unspecified & 26 & Male & Asian & Non-Spanish/non-Hispanic \\
\hline Patient 2 & Intimal Sarcoma & Primary tumor & 20 & Male & White & Non-Spanish/non-Hispanic \\
\hline Patient 3 & Intimal Sarcoma & Metastasis site unspecified & 42 & Male & White & Non-Spanish/non-Hispanic \\
\hline Patient 4 & Intimal Sarcoma & Primary tumor & 47 & Female & White & Non-Spanish/non-Hispanic \\
\hline Patient 5 & Intimal Sarcoma & Primary tumor & 46 & Female & Asian & Non-Spanish/non-Hispanic \\
\hline Patient 6 & Intimal Sarcoma & Primary tumor & 18 & Female & White & Spanish/Hispanic \\
\hline Patient 7 & Intimal Sarcoma & Primary tumor & 34 & Male & White & Non-Spanish/non-Hispanic \\
\hline Patient 8 & Intimal Sarcoma & Primary tumor & 69 & Female & White & Non-Spanish/non-Hispanic \\
\hline Patient 9 & Intimal Sarcoma & Metastasis site unspecified & 76 & Female & White & Non-Spanish/non-Hispanic \\
\hline Patient 10 & Intimal Sarcoma & Primary tumor & 56 & Female & White & Non-Spanish/non-Hispanic \\
\hline Patient 11 & Intimal Sarcoma & Primary tumor & 46 & Male & White & Non-Spanish/non-Hispanic \\
\hline Patient 12 & Intimal Sarcoma & Primary tumor & 71 & Female & White & Non-Spanish/non-Hispanic \\
\hline Patient 13 & Intimal Sarcoma & Primary tumor & 49 & Female & Asian & Unknown \\
\hline
\end{tabular}

Copy number alterations were available for 12 patients (see Figure 2). We identified amplifications in MDM2 $(n=9,75 \%)$, CDK4 $(n=6,50 \%)$, TERT $(n=6,50 \%), \operatorname{KIT}(n=4,33 \%), \operatorname{KDR}(n=3,25 \%)$, PDGFRA $(n=4,33 \%)$, NOTCH2 $(n=2,17 \%)$, ERBB3 $(n=3,25 \%)$, and GLI1 $(n=3,25 \%)$. Copy number losses in CDKN2A and CDKN2B were observed in three tumors (25\%).

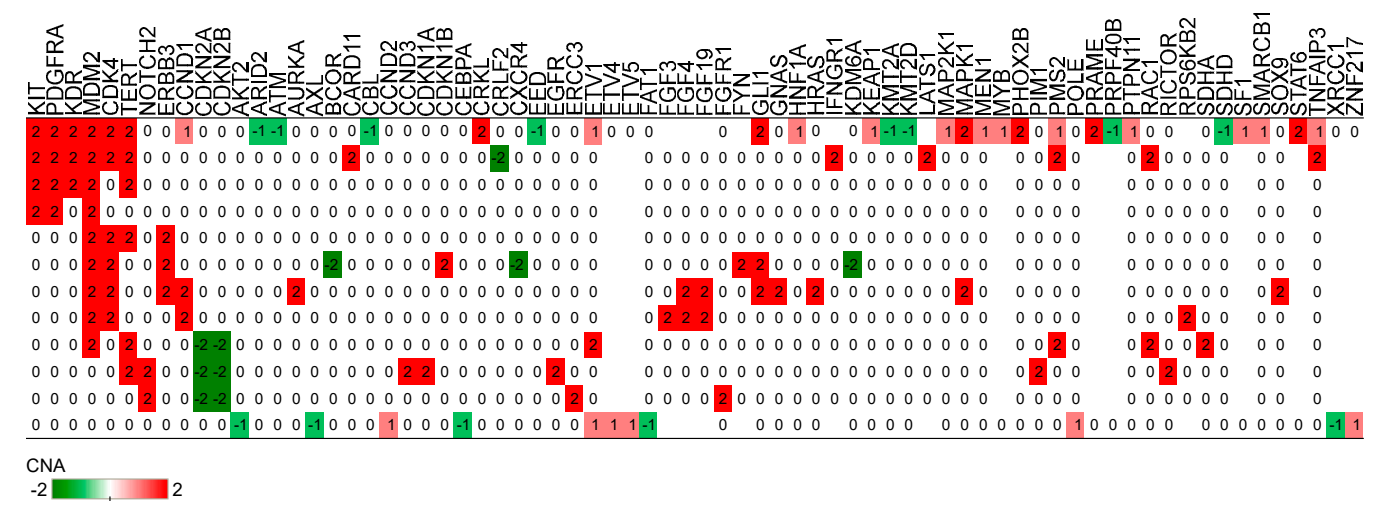

Figure 2. Copy number alterations in intimal sarcoma. Genes are shown in columns for patients in rows. Red color represents copy number gain while green denotes loss. The numbers show the copy number alteration (CNA) values from AACR GENIE. 
Next-generation sequencing revealed 38 somatic nonsynonymous mutations (33 missense, four frame shift, one nonsense, and one splice region) in intimal sarcoma tumors from nine patients (see Table 2). Among these mutations, M772V (c.2314A > G) and D850V (c.2549A > T) were found in $P D G F R B$ from two different primary tumors. The primary tumor sample with the D850V mutation in PDGFRB had concurrent mutations in PTCH1 (c.961C > A), ATR (c.4704C > G), and CDKN1A (c.419_420delGA). Potentially actionable genes are highlighted in green in Table 2.

Table 2. Somatic mutations in intimal sarcoma samples. Potentially actionable genes are highlighted in green. (Potentially actionable genes are highlighted in green.)

\begin{tabular}{|c|c|c|c|}
\hline Sample & Gene & Variant Classification & Mutation \\
\hline Patient 1 & ASXL1 & Missense & D864E \\
\hline Patient 1 & ASXL1 & Missense & G1299R \\
\hline Patient 1 & GLI1 & Missense & R171Q \\
\hline Patient 1 & IDH2 & Missense & I153V \\
\hline Patient 1 & KDR & Splice Region & \\
\hline Patient 1 & MDM2 & Intron & \\
\hline Patient 1 & MYD88 & Silent & D247D \\
\hline Patient 1 & NF1 & Missense & L121V \\
\hline Patient 1 & RARA & Intron & \\
\hline Patient 1 & RET & Missense & M1009T \\
\hline Patient 1 & SETBP1 & Missense & P1526Q \\
\hline Patient 2 & ARHGEF12 & Missense & E186K \\
\hline Patient 2 & KDM5A & Missense & S1408N \\
\hline Patient 2 & TMEM127 & Missense & I188V \\
\hline Patient 2 & TOPBP1 & Missense & L1499P \\
\hline Patient 3 & ERBB4 & Missense & H893R \\
\hline Patient 5 & BARD1 & Missense & A502N \\
\hline Patient 5 & ERCC5 & Missense & S453C \\
\hline Patient 5 & SOX2 & Missense & A263E \\
\hline Patient 5 & TP53 & Missense & R273C \\
\hline Patient 5 & U2AF1 & Missense & G212A \\
\hline Patient 7 & ATR & Missense & D1568E \\
\hline Patient 7 & CDKN1A & Frame Shift Del & R140Qfs*56 \\
\hline Patient 7 & FAT1 & Missense & P1333L \\
\hline Patient 7 & PDGFRB & Missense & D850V \\
\hline Patient 7 & PTCH1 & Missense & L321I \\
\hline Patient 7 & RECQL4 & Missense & E37K \\
\hline Patient 8 & ALK & Missense & E1460Q \\
\hline Patient 8 & GATA1 & Missense & G165S \\
\hline Patient 8 & GRIN2A & Missense & R1309Q \\
\hline Patient 8 & INPP4A & Missense & P773S \\
\hline Patient 8 & JAK3 & Missense & W716R \\
\hline Patient 8 & NOTCH4 & Missense & A1439T \\
\hline Patient 8 & PAK7 & Frame Shift Del & P612Lfs*3 \\
\hline Patient 10 & PDGFRB & Missense & M772V \\
\hline Patient 11 & EIF1AX & Missense & G6R \\
\hline Patient 11 & ERCC2 & Missense & T49A \\
\hline Patient 11 & MDM4 & Missense & D173H \\
\hline Patient 11 & NSD1 & Nonsense & Q2274* \\
\hline Patient 11 & SETD2 & Frame Shift Del & V1070Lfs $* 44$ \\
\hline Patient 12 & EP300 & Missense & S2404A \\
\hline Patient 12 & HGF & Frame Shift Ins & T4Gfs*39 \\
\hline Patient 13 & ATM & Intron & \\
\hline
\end{tabular}

Genomic rearrangement events included only a PDE4DIP-NOTCH2 and a MRPS30-ARID2 fusion (see Table 3). Interestingly, co-occurring alterations included a NOTCH2 copy number gain in the PDE4DIP-NOTCH2 fusion tumor and PDGFRB mutations in both fusion-positive cases. 
Table 3. Gene fusions in intimal sarcoma. Comments are from the GENIE database.

\begin{tabular}{|c|c|c|c|c|c|}
\hline Sample & Fusion & DNA Support & $\begin{array}{c}\text { RNA } \\
\text { Support }\end{array}$ & Frame & Comments \\
\hline Patient 7 & PDE4DIP-NOTCH2 & yes & unknown & in frame & $\begin{array}{l}\text { Note: The PDE4DIP (NM_022359)-NOTCH2 } \\
\text { (NM_024408) rearrangement event is a deletion } \\
\text { which results in the fusion of PDE4DIP exon } 1 \\
\text { and NOTCH2 exons 27-34. Its functional } \\
\text { significance is undetermined. }\end{array}$ \\
\hline Patient 10 & MRPS30-ARID2 & yes & unknown & unknown & $\begin{array}{l}\text { ARID2 (NM_152641) rearrangement: } t(5 ; 12) \\
\text { (p12;q12) (chr5:g.44601610::chr12:g.46245800) } \\
\text { Note: The ARID2 rearrangement is a } \\
\text { translocation which results in the truncation of } \\
\text { ARID2 exons 15-21. One of the breakpoints is } \\
\text { within ARID2 exon15. }\end{array}$ \\
\hline
\end{tabular}

We sought to analyze clinical trial results, but of the 406 sarcoma patients enrolled in clinical trials there were no patients with intimal sarcoma in a large Phase 1 clinic at the University of Texas MD Anderson Cancer Center. We hypothesize this was due to exclusion based on the location of their tumors.

\section{Discussion}

There is a large amount of heterogeneity in the presentation, histology, and genomic profile of intimal sarcomas $[1-4,18]$. The molecular variability and aggressive nature of this cancer makes it a challenging entity to diagnose and treat. Case reports and series have shown conventional chemotherapy with limited success, and curative radical surgery prolonging survival to a little over a year [5,11-13]. To our knowledge, there are no prospective clinical trials for patients with intimal sarcoma. These patients with intimal sarcoma will need to be included in clinical trials to determine which patients would benefit from genomically-matched therapies.

Our study confirmed the recurrence of copy number gains in MDM2 and CDK4 that coincide with the location 12q12-15, and PDGFRA in 4q12, as described in prior studies. The amplification of these genes strongly suggests that PDGFR and MDM2 pathways are important for tumorigenesis in IS. MDM2 inhibitors under investigation have demonstrated responses in early phase clinical trials in MDM2 amplified soft tissue sarcomas (with or without CDK4 co-amplification) such as liposarcoma and synovial sarcoma [19]. Our data support the use of NGS on intimal sarcomas to identify MDM2 amplification and consideration for such trials. Thirty-three percent were found to have PDGFRA amplification, but this can be explained by the choice of biopsy site due to intratumoral heterogeneity [7]. New mutations in PDGFRB found in our study may have implications for tumorigenesis and therapeutic options as well.

We also found novel somatic mutations in another receptor kinase gene such as $A L K$, which is known to be mutated or amplified in various other cancers such as lymphomas and non-small cell lung cancers [20]. However, the ALK E1460Q mutation is outside the tyrosine kinase domain and may not be relevant clinically. Other mutations identified include ATM/ATR, and PTCH1, a tumor suppressor that is part of the hedgehog signaling pathway involved in tumorigenesis [21,22]. A cell checkpoint kinase mutation, which is upstream from p53 in the signaling pathway, was also seen. All of these genes could be potential targets for treatment.

Additional findings of interest include fusion proteins involving NOTCH2-PDE4DIP and ARID2-MRPS30 and copy number gains in NOTCH2. Irregular NOTCH2 signaling has been associated with the initiation and progression of multiple cancers including liver, brain, and gastric cancers along with lymphomas [23]. In addition to NOTCH2, mutations in ARID2, a possible tumor suppressor, are also found in hepatocellular carcinomas [24]. The counterparts of these fusion proteins, such as MRPS30 and PDE4DIP, may also be associated with an increased risk for breast cancer and leptomeningeal disease progression, respectively $[25,26]$. Interestingly, both fusion proteins were 
found in primary tumors, but their effect on the tumorigenesis of intimal sarcomas is unknown and will require further investigation.

Limitations of this study include the sample size, which given the rarity of disease and biopsy site is likely due to intratumoral heterogeneity. Intimal sarcoma patients are currently not enrolled in clinical trials due to the location of the tumors in major vasculature. Since we noted actionable alterations in MDM2, CDK4, PDGFRA, and NOTCH2, as well as copy number losses in CDKN2A and CDKN2B, our study has clinical implications. There are now three US FDA approved CDK4/6 inhibitors for breast cancer, Palbociclib (IBRANCE ${ }^{\circledR}$, Pfizer Inc.), ribociclib (Kisqali ${ }^{\circledR}$, Novartis Pharmaceuticals Corporation), and abemaciclib (VERZENIO ${ }^{\mathrm{TM}}$, Eli Lilly and Company). Given the multiple aberrations seen in the CDK pathway, CDK4/6 inhibitors may be an option in clinical trials or off-label based on molecular profiling data. In addition, some of these tumors may be responsive to drugs that are already approved for the treatment of sarcomas like Pazopanib (Votrient ${ }^{\circledR}$ ), which is a multi-kinase VEGF based inhibitor that has activity against the PDGFR pathway as well. Other novel TKIs and MDM2 antagonists should be evaluated as treatments for advanced IS. In addition, the role of ATM/ATR, NOTCH2, and PTCH1 should be studied as potential therapeutic targets. Investigators should be encouraged to enroll these patients with specific molecular aberrations to basket trials.

\section{Materials and Methods}

We queried the AACR Project Genomics Evidence Neoplasia Information Exchange (GENIE) database (version 5) [27]. The GENIE registry derives existing CLIA-/ISO-certified genomic data [28]. Published records in Pubmed/Medline were reviewed for genomic data from several different platforms. Retrospective records were abstracted to appraise the benefit of using a targeted therapy approach in a large Phase 1 clinic at the University of Texas MD Anderson Cancer Center.

Ethics Statement: All AACR GENIE project data has been de-identified using the HIPAA Safe Harbor Method. Analyses were retrospective and were performed in accordance with the AACR GENIE Human Subjects Protection and Privacy policy. Institutional Review Board (IRB) details are provided in the AACR GENIE Data Guide [28].

\section{Conclusions}

Comprehensive genomic profiling may be considered for intimal sarcoma, both to confirm diagnosis and to explore treatment options on clinical trials. Clinical trial enrollment should be considered for all intimal sarcomas, and further exploration of copy number changes and genetic alterations is needed for this relatively chemotherapy-resistant disease. A global multicenter prospective registry/trial would best determine the response of this rare cancer to targeted therapies.

Author Contributions: Conceptualization, J.R. and V.S.; methodology, J.R., A.P.C., J.A.L., R.G., and V.S.; investigation, J.R., A.K., A.P.C., J.A.L., R.G., V.R., R.C.P., S.S., and V.S.; resources, J.R., and V.S.; writing—original draft preparation, A.K. and J.R.; writing—review and editing, J.R., A.K., A.P.C., J.A.L., R.G., V.R., R.C.P., S.S., and V.S.

Funding: The University of Texas MD Anderson Cancer Center is supported by grant CA016672 from the National Institutes of Health.

Acknowledgments: The authors would like to acknowledge the American Association for Cancer Research and its financial and material support in the development of the AACR Project GENIE registry, as well as members of the consortium for their commitment to data sharing. Interpretations are the responsibility of study authors.

Conflicts of Interest: Vivek Subbiah receives research funding for clinical trials from Novartis, Bayer, GlaxoSmithKline, Nanocarrier, Vegenics, Celgene, Northwest Biotherapeutics, Berghealth, Incyte, Fujifilm, Pharmamar, D3, Pfizer, Multivir, Amgen, Abbvie, Alfa-sigma, Agensys, Boston Biomedical, Idera Pharma, Inhibrx, Exelixis, Blueprint medicines, Loxo oncology, Inhbrx, Takeda and Roche/ Genentech, National Comprehensive Cancer Network, NCI-CTEP and UT MD Anderson Cancer Center. Travel: Novartis, Pharmamar, ASCO, ESMO, Helsin, Incyte. 


\section{References}

1. Neuville, A.; Collin, F.; Bruneval, P.; Parrens, M.; Thivolet, F.; Gomez-Brouchet, A.; Terrier, P.; de Montpreville, V.T.; Le Gall, F.; Hostein, I.; et al. Intimal sarcoma is the most frequent primary cardiac sarcoma: Clinicopathologic and molecular retrospective analysis of 100 primary cardiac sarcomas. Am. J. Surg. Pathol. 2014, 38, 461-469. [CrossRef] [PubMed]

2. Dewaele, B.; Floris, G.; Finalet-Ferreiro, J.; Fletcher, C.D.; Coindre, J.M.; Guillou, L.; Hogendoorn, P.C.; Wozniak, A.; Vanspauwen, V.; Schöffski, P.; et al. Coactivated platelet-derived growth factor receptor \{alpha\} and epidermal growth factor receptor are potential therapeutic targets in intimal sarcoma. Cancer Res. 2010, 70, 7304-7314. [CrossRef] [PubMed]

3. Bode-Lesniewska, B.; Zhao, J.; Speel, E.J.; Biraima, A.M.; Turina, M.; Komminoth, P.; Heitz, P.U. Gains of 12q13-14 and overexpression of $\mathrm{mdm} 2$ are frequent findings in intimal sarcomas of the pulmonary artery. Virchows Arch. 2001, 438, 57-65. [CrossRef] [PubMed]

4. Ito, Y.; Maeda, D.; Yoshida, M.; Yoshida, A.; Kudo-Asabe, Y.; Nanjyo, H.; Izumi, C.; Yamamoto, F.; Inoue, M.; Shibata, H.; et al. Cardiac intimal sarcoma with PDGFRbeta mutation and co-amplification of PDGFRalpha and MDM2: An autopsy case analyzed by whole-exome sequencing. Virchows Arch. 2017, 471, 423-428. [CrossRef] [PubMed]

5. Van Dievel, J.; Sciot, R.; Delcroix, M.; Vandeweyer, R.O.; Debiec-Rychter, M.; Dewaele, B.; Cornillie, J.; Van Cann, T.; Meyns, B.; Schöffski, P. Single-Center Experience with Intimal Sarcoma, an Ultra-Orphan, Commonly Fatal Mesenchymal Malignancy. Oncol. Res. Treat. 2017, 40, 353-359. [CrossRef] [PubMed]

6. Sebenik, M.; Ricci, A., Jr.; DiPasquale, B.; Mody, K.; Pytel, P.; Jee, K.J.; Knuutila, S.; Scholes, J. Undifferentiated intimal sarcoma of large systemic blood vessels: Report of 14 cases with immunohistochemical profile and review of the literature. Am. J. Surg. Pathol. 2005, 29, 1184-1193. [CrossRef]

7. Tajima, S.; Takanashi, Y.; Takahashi, T.; Neyatani, H. Intimal sarcoma of the abdominal aorta with platelet-derived growth factor receptor alpha overexpression and amplification in mural invasive cells and pulmonary metastatic cells but not in intimal spreading cells. Pathol. Int. 2015, 65, 426-431. [CrossRef]

8. Zhang, H.; Macdonald, W.D.; Erickson-Johnson, M.; Wang, X.; Jenkins, R.B.; Oliveira, A.M. Cytogenetic and molecular cytogenetic findings of intimal sarcoma. Cancer Genet. Cytogenet. 2007, 179, 146-149. [CrossRef]

9. Fukuda, W.; Morohashi, S.; Fukuda, I. Intimal sarcoma of the pulmonary artery-Diagnostic challenge. Acta Cardiol. 2011, 66, 539-541. [CrossRef]

10. Anderson, M.B.; Kriett, J.M.; Kapelanski, D.P.; Tarazi, R.; Jamieson, S.W. Primary pulmonary artery sarcoma: A report of six cases. Ann. Thorac. Surg. 1995, 59, 1487-1490. [CrossRef]

11. Blackmon, S.H.; Rice, D.C.; Correa, A.M.; Mehran, R.; Putnam, J.B.; Smythe, W.R.; Walkes, J.C.; Walsh, G.L.; Moran, C.; Singh, H.; et al. Management of primary pulmonary artery sarcomas. Ann. Thorac. Surg. 2009, 87, 977-984. [CrossRef] [PubMed]

12. Tamborini, E.; Casieri, P.; Miselli, F.; Orsenigo, M.; Negri, T.; Piacenza, C.; Stacchiotti, S.; Gronchi, A.; Pastorino, U.; Pierotti, M.A.; et al. Analysis of potential receptor tyrosine kinase targets in intimal and mural sarcomas. J. Pathol. 2007, 212, 227-235. [CrossRef] [PubMed]

13. Shehatha, J.; Saxena, P.; Clarke, B.; Dunning, J.; Konstantinov, I.E. Surgical management of extensive pulmonary artery sarcoma. Ann. Thorac. Surg. 2009, 87, 1269-1271. [CrossRef] [PubMed]

14. Nakahira, A.; Ogino, H.; Sasaki, H.; Katakami, N. Long-term survival of a pulmonary artery sarcoma produced by aggressive surgical resection and adjuvant chemoradiotherapy. Eur. J. Cardiothorac. Surg. 2007, 32, 388-390. [CrossRef] [PubMed]

15. Zhao, J.; Roth, J.; Bode-Lesniewska, B.; Pfaltz, M.; Heitz, P.U.; Komminoth, P. Combined comparative genomic hybridization and genomic microarray for detection of gene amplifications in pulmonary artery intimal sarcomas and adrenocortical tumors. Genes Chromosomes Cancer 2002, 34, 48-57. [CrossRef] [PubMed]

16. Call, J.W.; Wang, Y.; Montoya, D.; Scherzer, N.J.; Heinrich, M.C. Survival in advanced GIST has improved over time and correlates with increased access to post-imatinib tyrosine kinase inhibitors: Results from Life Raft Group Registry. Clin. Sarcoma Res. 2019, 9, 019-0114.

17. Pestana, R.C.; Groisberg, R.; Roszik, J.; Subbiah, V. Precision Oncology in Sarcomas: Divide and Conquer. JCO Precision Oncol. 2019, 1-16. [CrossRef] 
18. Groisberg, R.; Hong, D.S.; Holla, V.; Janku, F.; Piha-Paul, S.; Ravi, V.; Benjamin, R.; Kumar Patel, S.; Somaiah, N.; Conley, A.; et al. Clinical genomic profiling to identify actionable alterations for investigational therapies in patients with diverse sarcomas. Oncotarget 2017, 8, 39254-39267. [CrossRef]

19. Bauer, T.M.; Gounder, M.M.; Weise, A.M.; Schwartz, G.K.; Carvajal, R.D.; Kumar, P.; Zernovak, O.; Beck, A.; Doyle, J.; Mendell-Harary, J.; et al. A phase 1 study of MDM2 inhibitor DS-3032b in patients with well/de-differentiated liposarcoma (WD/DD LPS), solid tumors (ST) and lymphomas (L). J. Clin. Oncol. 2018, 36, 11514. [CrossRef]

20. Ardini, E.; Magnaghi, P.; Orsini, P.; Galvani, A.; Menichincheri, M. Anaplastic Lymphoma Kinase: Role in specific tumours, and development of small molecule inhibitors for cancer therapy. Cancer Lett. 2010, 299, 81-94. [CrossRef]

21. Yang, J.; Yu, Y.; Hamrick, H.E.; Duerksen-Hughes, P.J. ATM, ATR and DNA-PK: Initiators of the cellular genotoxic stress responses. Carcinogenesis 2003, 24, 1571-1580. [CrossRef] [PubMed]

22. Adolphe, C.; Hetherington, R.; Ellis, T.; Wainwright, B. Patched1 functions as a gatekeeper by promoting cell cycle progression. Cancer Res 2006, 66, 2081-2088. [CrossRef] [PubMed]

23. Xiu, M.X.; Liu, Y.M. The role of oncogenic Notch2 signaling in cancer: A novel therapeutic target. Am. J. Cancer Res. 2019, 9, 837-854. [PubMed]

24. Li, M.; Zhao, H.; Zhang, X.; Wood, L.D.; Anders, R.A.; Choti, M.A.; Pawlik, T.M.; Daniel, H.D.; Kannangai, R.; Offerhaus, G.J.; et al. Inactivating mutations of the chromatin remodeling gene ARID2 in hepatocellular carcinoma. Nat. Genet. 2011, 43, 828-829. [CrossRef] [PubMed]

25. Guo, X.; Lin, W.; Bao, J.; Cai, Q.; Pan, X.; Bai, M.; Yuan, Y.; Shi, J.; Sun, Y.; Han, M.R.; et al. A Comprehensive cis-eQTL Analysis Revealed Target Genes in Breast Cancer Susceptibility Loci Identified in Genome-wide Association Studies. Am. J. Hum. Genet. 2018, 102, 890-903. [CrossRef] [PubMed]

26. Li, Y.; Liu, B.; Connolly, I.D.; Kakusa, B.W.; Pan, W.; Nagpal, S.; Montgomery, S.B.; Hayden Gephart, M. Recurrently Mutated Genes Differ between Leptomeningeal and Solid Lung Cancer Brain Metastases. J. Thorac. Oncol. 2018, 13, 1022-1027. [CrossRef]

27. AACR Project GENIE Consortium. AACR Project GENIE: Powering Precision Medicine through an International Consortium. Cancer Discov. 2017, 7, 818-831. [CrossRef]

28. AACR GENIE Data Guide. Available online: https://www.aacr.org/Research/Research/Documents/20190713 GENIE_Data_Guide_6.1-public_final.pdf (accessed on 15 August 2019). 